The Astrophysical Journal, 486:230-241, 1997 September 1

(C) 1997. The American Astronomical Society. All rights reserved. Printed in U.S.A.

\title{
THE DYNAMICS OF THE M87 GLOBULAR CLUSTER SYSTEM ${ }^{1}$
}

\author{
Judith G. COHEN ${ }^{2}$ AND ANTON Ryzhov ${ }^{2,3}$ \\ Received 1997 January 24; accepted 1997 April 3
}

\begin{abstract}
We present the results from a study of the dynamics of the system of globular clusters around M87. After eliminating foreground galactic stars and background galaxies, we end up with a sample of 205 bona fide M87 globular clusters for which we have radial velocities determined from multislit spectra taken with the Low Resolution Imaging Spectrograph on the Keck Telescope. We find that the mean radial velocity of the M87 globular clusters agrees well with that of M87 itself and that the velocity histogram is well represented by a Gaussian distribution. We find evidence for rotation in the globular cluster system. We find that the observed velocity dispersion of the M87 globular cluster system increases with radius from $270 \mathrm{~km} \mathrm{~s}^{-1}$ at $r=9 \mathrm{kpc}$ to $\approx 400 \mathrm{~km} \mathrm{~s}^{-1}$ at $r=40 \mathrm{kpc}$. The inferred massto-light ratio in solar units increases from 5 at $r=9 \mathrm{kpc}$ to $\approx 30$ at $r=40 \mathrm{kpc}$ with $M(r) \sim r^{1.7}$. The long-slit optical spectroscopy near the center of M87 and the recent analysis of the ROSAT X-ray data are in good agreement with this analysis near the nucleus and in the outer parts of M87, respectively.

Subject headings: galaxies: halos — galaxies: individual (M87) — galaxies: kinematics and dynamics galaxies: star clusters
\end{abstract}

\section{INTRODUCTION}

The existence of dark matter around spiral galaxies was established by studies of their rotation curves at large radii using both $21 \mathrm{~cm}$ (Rogstad \& Shostak 1972) and optical (Rubin et al. 1985) emission lines from their gaseous disks. As discussed in the review by de Zeeuw \& Franx (1991), for example, elliptical galaxies in general do not have much gas, and it has been difficult to find any evidence for the possible presence of massive halos around them.

Globular clusters are ideal probes for studying the dynamics of the outer parts of galaxies. Their masses can be ignored compared to the mass of the galaxy. Except in the Galaxy, their distances from the galactic center and their orbits cannot be determined directly, but they are plentiful enough around some galaxies that statistics can be used to overcome this limitation. One such case is M87 at the center of the Virgo Cluster, which has an extremely rich globular cluster system, with thousands of members. Four meter class telescopes are just large enough to reach the brightest of these spectroscopically, and they have been studied by Mould, Oke, \& Nemec (1987), Huchra \& Brodie (1987), and Mould et al. (1990). Among the brightest objects, contamination by foreground galactic stars is severe and this reduces the efficiency of isolating true M87 globular clusters from among the candidates.

Mould et al. (1990) assembled the data available at that time, combining the Palomar and MMT samples to get a total sample of 43 globular clusters in M87. Grillmair et al. (1994) have assembled a sample of similar size for globular clusters in NGC 1399, the cD galaxy in the Fornax cluster of galaxies. But because of the small sample size, their estimates of the many critical kinematic parameters of these

\footnotetext{
${ }^{1}$ Based in large part on observations obtained at the W. M. Keck Observatory, which is operated jointly by the California Institute of Technology and the University of California.

2 Palomar Observatory, Mail Stop 105-24, California Institute of Technology, Pasadena, CA 91125.

${ }^{3}$ Current address: Department of Physics, Box 351560, University of Washington, Seattle, WA 98195-1560.
}

globular cluster systems, including the rotation and the velocity dispersion as a function of radius, are of low accuracy. Merritt \& Tremblay (1993) estimated from numerical simulations that a sample of $\approx 200$ globular clusters in M87 is required to derive accurately the most fundamental parameter of the mass distribution of the halo of M87, the exponent of the power law for the radial mass profile.

We present here a study of the kinematics of the globular clusters around M87 with the goal of probing the distribution of mass around this galaxy at the center of the Virgo Cluster, deducing the mass-to-light ratio in the halo of M87, and comparing our results to inferences from recent X-ray missions and from recent optical spectroscopy of the stellar component of M87. With the light-gathering power of the $10 \mathrm{~m}$ Keck I Telescope coupled with an efficient multiobject spectrograph, we have succeeded in overcoming the limitations of earlier work and have accumulated a sample of more than 200 globular clusters in M87 with accurate radial velocities.

A study of the abundances and ages of the M87 globular clusters that can also be inferred from our data set will follow shortly.

\section{THE SAMPLE OF M87 GLOBULAR CLUSTERS}

The candidates were chosen from the photographic survey of Strom et al. (1981) for globular clusters in M87. This survey of objects with $B<23.5 \mathrm{mag}$ covers a square region about $14^{\prime}$ on a side. The central region of M87 $\left(R<60^{\prime \prime}\right)$ was excluded, presumably owing to saturation of the photographic plates, and the candidate list is incomplete out to $R<90^{\prime \prime}$. There are 1728 objects in their sample. Our sample includes the 723 objects with $B<22.5 \mathrm{mag}$ from the Strom et al. survey. We have found that their object positions are adequate for designing slit masks that align satisfactorily.

A sample of bright globular cluster candidates near the center of M87 was added to fill in the central region. The radial distances from the center of M87 of these added 
TABLE 1

LOCATION OF AdDitional Globular Clusters Near the Center of M87

\begin{tabular}{lrr}
\hline \hline ID Number & $\begin{array}{c}\text { East }^{\mathrm{a}} \\
(\mathrm{arcsec})\end{array}$ & $\begin{array}{r}\text { North }^{\mathrm{a}} \\
(\mathrm{arcsec})\end{array}$ \\
\hline $5001 \ldots \ldots \ldots$ & +47.8 & -99.1 \\
$5002 \ldots \ldots \ldots$ & +37.9 & -97.0 \\
$5003 \ldots \ldots \ldots$ & +24.2 & -96.1 \\
$5005 \ldots \ldots \ldots$ & +34.7 & -85.7 \\
$5008 \ldots \ldots \ldots$ & +51.6 & -75.8 \\
$5010 \ldots \ldots \ldots$ & -11.8 & -55.9 \\
$5012 \ldots \ldots \ldots$ & +60.6 & -99.1 \\
$5014 \ldots \ldots \ldots$ & +15.8 & -24.6 \\
$5015 \ldots \ldots \ldots$ & +34.3 & -21.8 \\
$5016 \ldots \ldots \ldots$ & +46.3 & -42.0 \\
$5017 \ldots \ldots \ldots$ & +50.5 & -50.7 \\
$5020 \ldots \ldots \ldots$ & -24.4 & -51.4 \\
$5021 \ldots \ldots \ldots$ & +22.9 & -117.8 \\
$5024 \ldots \ldots \ldots$ & -15.4 & -8.4 \\
$5025 \ldots \ldots \ldots$ & -22.5 & -61.9 \\
$5026 \ldots \ldots \ldots$ & -22.1 & -7.3 \\
$5028 \ldots \ldots \ldots$ & +64.5 & -19.5 \\
\hline \multicolumn{3}{c}{ a Offsets with respect to the M87 } \\
globular cluster Strom 928.
\end{tabular}

objects range from $24^{\prime \prime}$ to $71^{\prime \prime}$. They were chosen from direct images taken with the Low Resolution Imaging Spectrograph (LRIS; Oke et al. 1995) on the $10 \mathrm{~m}$ Keck I Telescope and their positions relative to the bright globular cluster Strom 928, which is itself 23.2 west and 62 ".3 north of the nucleus of M87, are given in Table 1.

Throughout this paper, we use the Strom et al. (1981) running numbers to identify the clusters, while the additional objects near the center of M87 are denoted with running numbers beginning with 5001 .

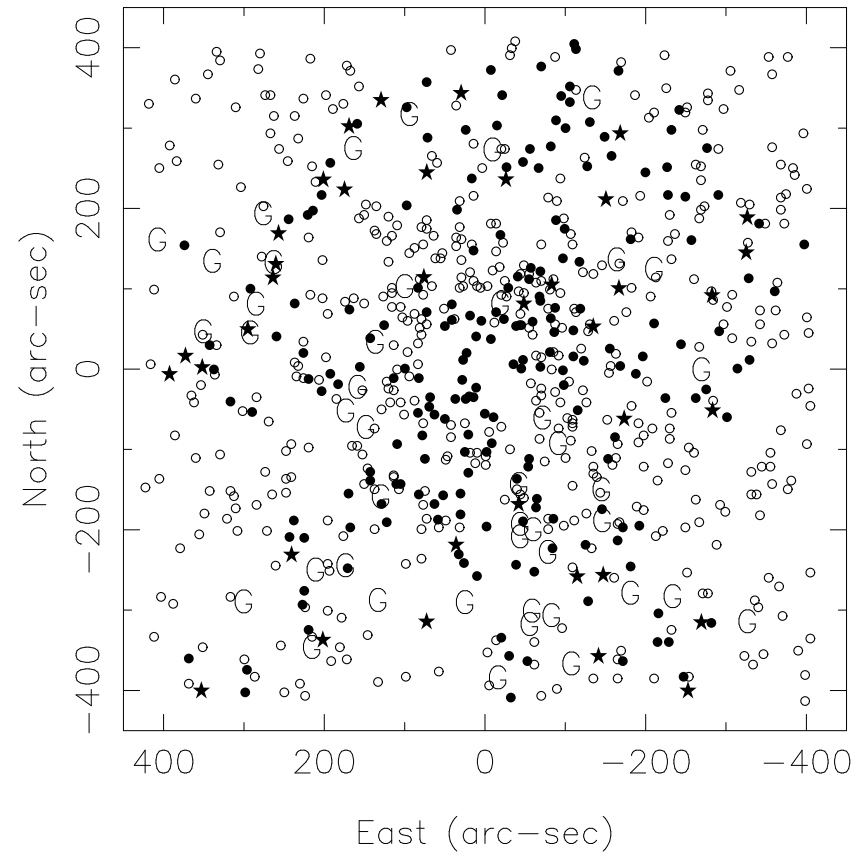

FIG. 1.-Spatial distribution of the sample of candidate M87 globular clusters from the Strom et al. (1981) survey is shown. Objects for which $v_{r}$ were determined are indicated by filled dots. Galactic stars are shown as filled asterisks, while galaxies are indicated by "G."
TABLE 2

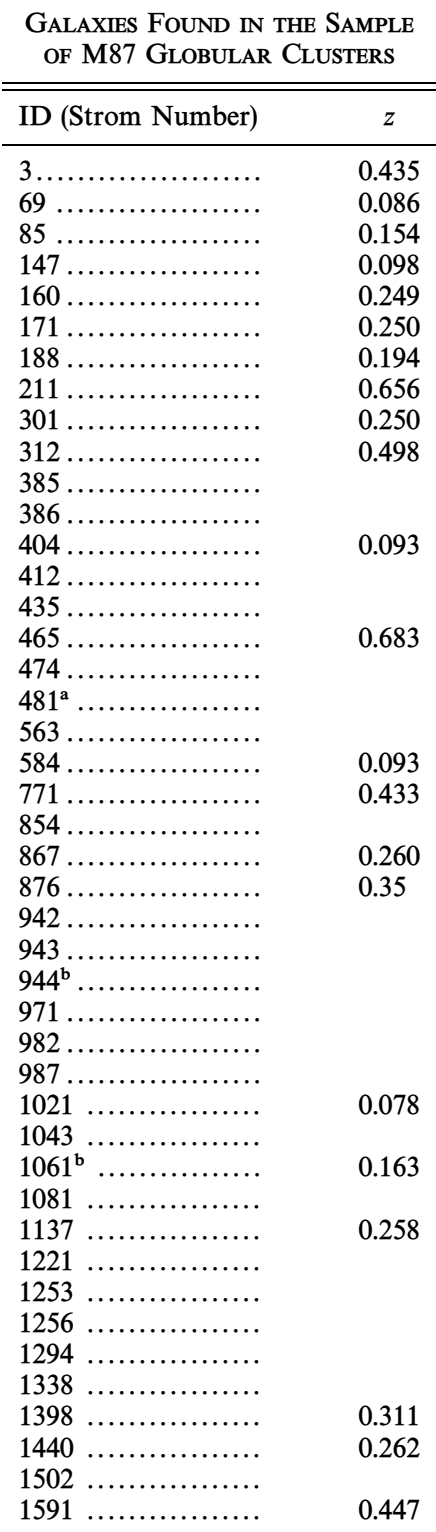

${ }^{\text {a }}$ The spectrum of this object shows broad emission lines.

b This object consists of a galaxy with a nearby stellar object.

About half of the objects in the list of candidates defined above were actually observed. For each slit mask, the first selection criterion was that the location of the candidate be close to the center line of the slit mask in the LRIS focal plane, while the second one was brightness. The few very bright objects were avoided, as they are mostly galactic stars, while objects in the faintest $0.5 \mathrm{mag}$ of the sample were not included in slit masks unless no other suitable candidate was available near the desired position in the sky.

The spectra of about $15 \%$ of the objects in these 14 slit masks were too noisy, and no features could be recognized; hence, no radial velocity could be deduced.

Fourteen M dwarfs (Strom 38, 87, 246, 354, 670, 681, 782, 801, 937, 996, 1549, 1551, 1608 and 1610) and one white dwarf (Strom 178) were found. These are assumed to be galactic stars and are not considered further.

The sample of candidate globular clusters in M87 turns out to be badly contaminated by galaxies, particularly in 
the outer parts of M87. Whenever possible, to eliminate background galaxies, direct images taken with LRIS were used to verify that candidate M87 globulars had images that were not extended. Such LRIS direct images, particularly for the outermost parts of M87, were not always available before the masks had to be designed. Table 2 lists the 44 galaxies that were found. If no redshift is given, the galaxy was identified as such from LRIS direct images.

Figure 1 shows the distribution on the sky of the objects with measured radial velocities, including the galactic stars and the galaxies, as well as that of the entire $B<22.5 \mathrm{mag}$ sample from Strom's lists.

\section{OBSERVATIONS AND DATA ANALYSIS}

We have used the LRIS on the Keck I Telescope in the multislit mode. Slit masks whose length was 7.3 were used to maximize the efficiency of observations, with an average of 28 objects per mask. Two or three $3000 \mathrm{~s}$ exposures were obtained for each slit mask. All the observations used $600 \mathrm{~g}$ $\mathrm{mm}^{-1}$ gratings (dispersion $1.24 \AA \mathrm{pixel}^{-1}$ ) with $1^{\prime \prime} 4$ wide slits giving an effective resolution of $8.1 \AA$ (ignoring anamorphic magnification, which improves this somewhat, especially in the red). The detector is a $2048 \times 2048$ pixel Tektronix CCD, so the spectral coverage is $\approx 2500 \AA$.

These observations were carried out on 7 nights over a 3 yr period. The seeing near the zenith for these nights ranged from 0 ".6 to 1"2.

A total of 14 different slit masks have been used. In 1994, four masks were used with the $600 \mathrm{~g} \mathrm{~mm}^{-1}$ grating blazed at $7500 \AA$ centered at $8400 \AA$. The dominant feature in these spectra is the infrared Ca triplet. In early 1994, the detector electronics were noisier than they should have been, a situation corrected in mid-1994. In 1995, eight masks were used with that same grating centered at $5500 \AA$ so as to pick up the $\mathrm{Mg}$ triplet and nearby strong $\mathrm{Fe}$ blends, as well as the Balmer lines $\mathrm{H} \alpha$ and $\mathrm{H} \beta$. These masks have a position angle of $0^{\circ}-315^{\circ}$ in $45^{\circ}$ increments. Each mask is laid out radially along its selected position angle, with one end of the effective slit placed close to the nucleus of M87 near the inner radial limit of the Strom et al. (1981) survey. In 1996, a new $600 \mathrm{~g} \mathrm{~mm}^{-1}$ grating became available with a bluer blaze angle (5000 $)$. This was used with two masks centered on the nucleus of M87. The exposures here were only $2500 \mathrm{~s}$ long, with two or three exposures per slit mask. These spectra were again centered at $5500 \AA$. With the exception of the 1996 spectra, all these observations used dual amplifier readouts to maximize the efficiency of observing. Since these are multislit spectra, the actual wavelength coverage for a particular object depends on its position with respect to the center of the entrance field of the LRIS.

The LRIS has some flexure. In part because of this, the spectra were not flat-fielded, although they were corrected for the difference in bias level and gain between the two amplifiers used in the dual amplifier readout. After this, the cosmic rays were removed. Then, the individual slitlet spectra were cut out of the full CCD frame. Next, the twodimensional spectrum of each slitlet was corrected for distortions both along and perpendicular to the dispersion, arising partially from distortions in the LRIS camera as well as from the long slit length used by LRIS slit masks. A second-order polynomial was fitted to the sky across each pixel in the dispersion direction, the sky was removed, and the appropriate sum along the slit yielded the spectrum for each object. The one-dimensional spectra from the various exposures of a given object with a given mask were summed to get the final result for each candidate.

The dominant problem in these spectra (at least after mid-1994, when the detector electronics were fixed) is the nonuniformity of the width of the slit. The slits are punched by a numerically controlled machine and need to be carefully cleaned of chips prior to use. Variations of a few percent in width are common, variations of $5 \%$ occasionally occur, and even larger variations occur if the masks are not cleaned properly. Efforts are underway within the California Association for Research in Astronomy to reduce or eliminate this problem. Correction of this variation of slit width is not straightforward given the flexure and was not incorporated into our analysis scripts.

To ensure the best possible accuracy, the wavelength calibration was carried out using the most unblended night-sky emission lines within the spectra of the globular clusters themselves. The region of the infrared $\mathrm{Ca}$ triplet is full of these, and there the wavelength calibration is very good. Typical fits (a third-order polynomial suffices) including 14 night-sky lines distributed along the length of the spectrum have a $1 \sigma \mathrm{rms}$ residual in the fit of $0.2 \AA\left(7 \mathrm{~km} \mathrm{~s}^{-1}\right)$. The $5500 \AA$ region has far fewer lines both in the night-sky spectrum (no Hg lines are seen from Mauna Kea) and in the available arc spectra. The $5199 \AA$ night-sky emission line is the last detectable night-sky emission feature on the blue side of these spectra. Its presence was crucial to anchor the wavelength scale. When the background of the integrated stellar light of M87 is very bright, this weak emission line is sometimes lost. In a very few cases, for $\lambda<5577 \AA$, the wavelength scale at the blue end had to be determined from other spectra with better arc coverage. The wavelength scale of each exposure of each multislit was independently calibrated.

All the data analysis was carried out using Figaro.

The spectra of the M87 globular clusters show no sign of spatial or velocity resolution. The "sky" spectra, consisting of the night sky and the halo light from M87 itself, show obvious line broadening for those globular clusters close to the center of M87, presumably owing to the velocity dispersion of the M87 stars.

\section{RADIAL VELOCITIES}

These spectra of candidate globular clusters in M87 cover one of two different wavelength regions. The radial velocities for the spectra centered at $8500 \AA$ were obtained by averaging those inferred from $\mathrm{H} \alpha$, which occasionally fell within the wavelength regime covered, and from each of the detected components of the infrared Ca triplet. (Only for the brightest M87 globular clusters was the weakest of the triplet lines clearly detected.) The radial velocities for the spectra centered at $5500 \AA$ were obtained via averaging the results of cross-correlations covering the region from 5150 to $5400 \AA$ and also the $\mathrm{H} \alpha$ region. $\mathrm{H} \alpha$ is outside the wavelength region covered in about $25 \%$ of these spectra. While $\mathrm{H} \beta$ is often included, the wavelength scale at $4900 \AA$ is poorly determined. The brightest M87 globular cluster in the particular mask being reduced was used as a template for the spectra from that mask. Its radial velocity was determined using the laboratory wavelengths of the $\mathrm{Mg}$ triplet, of the adjacent strong Fe blends, and of $\mathrm{H} \alpha$. The crosscorrelations were checked by visual inspection of the spectra, as sometimes spikes from cosmic rays throw the results off, and these had to be removed manually from the 
spectra or the radial velocity determined via eye estimates of the line centers.

Table 3 lists the heliocentric radial velocity for 229 candidate globular clusters in M87 in our sample. The histogram of heliocentric radial velocities for the candidate M87 globular clusters is shown in Figure 2. It is double peaked, with the majority of the objects distributed about the radial velocity of M87, while about $10 \%$ of the objects reside in a narrower peak centered on $0 \mathrm{~km} \mathrm{~s}^{-1}$. Even though the $\mathrm{M}$ dwarfs have already been excluded, substantial contamination by galactic stars is still present. Since it is impossible to separate the galactic stars of spectral type K from M87 globular clusters within this peak, we reject all objects with $v_{r}<250 \mathrm{~km} \mathrm{~s}^{-1}$, almost all of which are undoubtedly galactic stars. This brings the number of objects in our sample down to 205. To avoid skewing the distributions, we must also reject objects in the corresponding interval of the highvelocity tail of the $v_{r}$ distribution. However, there are no objects with such large positive radial velocities in our sample.

These 205 globular clusters in M87 with kinematic data from our LRIS observations are the sample used in the remainder of this paper.

\subsection{Uncertainty of the Radial Velocities}

The accuracy of the radial velocities depends on many factors, including the signal in the spectrum of the object, the signal in the spectrum of the background, the strength of the spectral features for each object, the accuracy of the background subtraction algorithm used, etc. The signal in the spectrum of the object depends on the transparency during the observations, the integration time $(6000-9000 \mathrm{~s}$ total), and the accuracy of alignment of the object with the slitlet in the slit mask. If the object is too close to the upper or lower edge of the slitlet, sky subtraction becomes more difficult.

Another potential source of error is illumination effects. In normal long-slit spectra, the radial velocity for a point

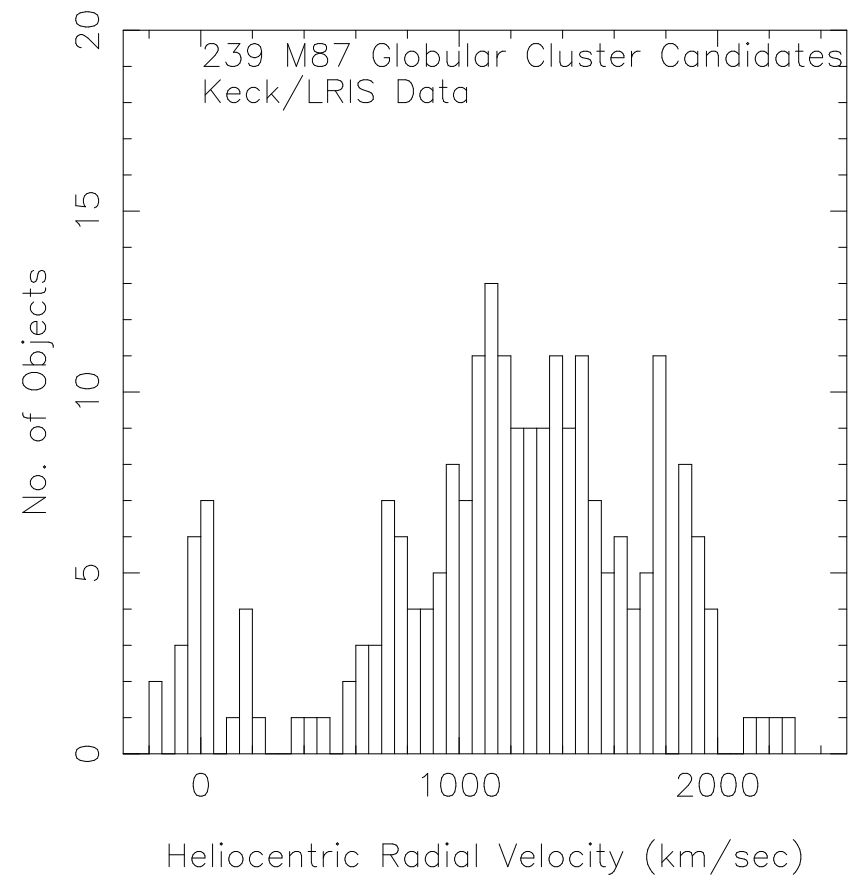

FIG. 2.-Histogram of the heliocentric radial velocities for the candidate M87 globular clusters. Galaxies and M stars have been excluded.
TABLE 3

RADIAL VELOCITIES FOR

CANDIDATE GLOBULAR Clusters in M87

\begin{tabular}{|c|c|}
\hline $\mathrm{ID}^{\mathrm{a}}$ & $\begin{array}{c}v_{r}{ }^{\mathrm{b}} \\
\left(\mathrm{km} \mathrm{s}^{-1}\right)\end{array}$ \\
\hline $5001 \ldots$ & 929 \\
\hline $5002 \ldots$ & 1252 \\
\hline $5003 \ldots$ & 1294 \\
\hline $5005 .$. & 1178 \\
\hline $5008 \ldots$ & 195 \\
\hline $5010 \ldots$ & 1401 \\
\hline $5012 \ldots$ & 1718 \\
\hline $5014 \ldots$ & 1278 \\
\hline $5015 \ldots$ & 1669 \\
\hline n............ & 1478 \\
\hline $5017 \ldots$ & 1173 \\
\hline $5020 \ldots$ & 1646 \\
\hline $5021 \ldots$ & 1767 \\
\hline $5024 \ldots$ & 1076 \\
\hline $5025 \ldots$ & 1762 \\
\hline $5026 \ldots$ & 1990 \\
\hline $5028 \ldots$ & 1414 \\
\hline 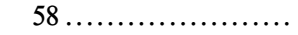 & 1923 \\
\hline , n.......... & -28 \\
\hline $66 \ldots$ & 2260 \\
\hline $91 \ldots$ & 179 \\
\hline $101 \ldots$ & 1332 \\
\hline $107 \ldots$ & 1515 \\
\hline $141 \ldots$ & 1115 \\
\hline $176 \ldots$ & 2210 \\
\hline 177 & 1629 \\
\hline $186 \ldots$ & 1761 \\
\hline ............. & 716 \\
\hline $235 \ldots$ & 31 \\
\hline $248 \ldots$ & 1016 \\
\hline $252 \ldots$ & -41 \\
\hline $279 \ldots$ & 820 \\
\hline $280 \ldots$ & 1044 \\
\hline $286 \ldots$ & 12 \\
\hline $290 \ldots$ & 1413 \\
\hline $292 \ldots$ & 847 \\
\hline n........... & 1219 \\
\hline $311 \ldots$ & 781 \\
\hline $313 \ldots$ & 1768 \\
\hline $314 \ldots$ & 1236 \\
\hline .............. & 1376 \\
\hline $323 \ldots$ & 1124 \\
\hline $324 \ldots$ & 359 \\
\hline $348 \ldots$ & 817 \\
\hline ............ & 736 \\
\hline $350 \ldots$ & 1244 \\
\hline $357 \ldots$ & -86 \\
\hline $376 \ldots$ & 1182 \\
\hline .................. & 1978 \\
\hline $395 \ldots \ldots \ldots \ldots \ldots \ldots$ & 1890 \\
\hline …............... & 15 \\
\hline n........... & 1910 \\
\hline $418 \ldots$ & 1866 \\
\hline ............ & -153 \\
\hline $421 \ldots \ldots$ & 1724 \\
\hline $423 \ldots$ & 1081 \\
\hline 442 . & 1424 \\
\hline 453 & 1980 \\
\hline $490 \ldots$ & 1570 \\
\hline $491 \ldots \ldots$ & 1069 \\
\hline $492 \ldots$ & 1498 \\
\hline $518 \ldots$ & 42 \\
\hline n........... & 1315 \\
\hline $526 \ldots \ldots$ & 1169 \\
\hline $537 \ldots$ & 1467 \\
\hline $571 \ldots$ & 1757 \\
\hline ............ & 999 \\
\hline ............. & 1507 \\
\hline $588 \ldots$ & 1637 \\
\hline ............. & 555 \\
\hline
\end{tabular}


TABLE 3-Continued

\begin{tabular}{|c|c|}
\hline $\mathrm{ID}^{\mathrm{a}}$ & $\begin{array}{c}v_{r}^{\mathrm{b}} \\
\left(\mathrm{km} \mathrm{s}^{-1}\right)\end{array}$ \\
\hline $611 \ldots$ & 1319 \\
\hline $614 \ldots$ & 1891 \\
\hline n.......... & 1760 \\
\hline 647 . & 972 \\
\hline 649. & 1373 \\
\hline$\ldots \ldots \ldots \ldots$ & 2110 \\
\hline n.......... & 1563 \\
\hline$\ldots \ldots \ldots \ldots$ & 702 \\
\hline $678 \ldots$ & -94 \\
\hline$\ldots \ldots \ldots \ldots$ & 1218 \\
\hline n.......... & 1793 \\
\hline$\ldots \ldots \ldots \ldots$ & 784 \\
\hline$\ldots \ldots \ldots \ldots$ & 1869 \\
\hline$\ldots \ldots \ldots \ldots$ & 1203 \\
\hline$\ldots \ldots \ldots \ldots$ & 1315 \\
\hline n.......... & 467 \\
\hline ........... & 1365 \\
\hline$\ldots \ldots \ldots \ldots$ & 1212 \\
\hline$\ldots \ldots \ldots \ldots$ & 1266 \\
\hline$\ldots \ldots \ldots \ldots$ & 1406 \\
\hline$\ldots \ldots \ldots \ldots$ & 1481 \\
\hline n.......... & 1891 \\
\hline$\ldots \ldots \ldots \ldots$ & 1097 \\
\hline ............ & 1130 \\
\hline$\ldots \ldots \ldots \ldots$ & 950 \\
\hline $801 \ldots \ldots \ldots \ldots \ldots \ldots \ldots$ & -30 \\
\hline$\ldots \ldots \ldots \ldots \ldots$ & 612 \\
\hline$\ldots \ldots \ldots \ldots$ & 1436 \\
\hline$\ldots \ldots \ldots \ldots \ldots$ & 1331 \\
\hline $824 \ldots \ldots \ldots \ldots \ldots \ldots \ldots$ & 1212 \\
\hline$\ldots \ldots \ldots \ldots$ & 1109 \\
\hline n.......... & 1477 \\
\hline n.......... & 1148 \\
\hline$\ldots \ldots \ldots \ldots \ldots$ & 1086 \\
\hline$\ldots \ldots \ldots \ldots \ldots$ & 1650 \\
\hline$\ldots \ldots \ldots \ldots$ & 759 \\
\hline $868 \ldots \ldots \ldots \ldots \ldots \ldots \ldots$ & 1480 \\
\hline $871 \ldots \ldots \ldots \ldots \ldots \ldots \ldots$ & 1073 \\
\hline $881 \ldots \ldots \ldots \ldots \ldots \ldots \ldots$ & 796 \\
\hline $887 \ldots \ldots \ldots \ldots \ldots \ldots$ & 1778 \\
\hline $892 \ldots \ldots \ldots \ldots \ldots \ldots \ldots$ & 1879 \\
\hline $902 \ldots \ldots \ldots \ldots \ldots \ldots$ & 1587 \\
\hline $904 \ldots \ldots \ldots \ldots \ldots \ldots \ldots$ & 914 \\
\hline $910 \ldots \ldots \ldots \ldots \ldots \ldots \ldots$ & 1007 \\
\hline $917 \ldots \ldots \ldots \ldots \ldots \ldots \ldots$ & 882 \\
\hline $922 \ldots \ldots \ldots \ldots \ldots \ldots \ldots$ & 1760 \\
\hline $928 \ldots \ldots \ldots \ldots \ldots \ldots \ldots$ & 1327 \\
\hline $937 \ldots \ldots \ldots \ldots \ldots \ldots$ & 930 \\
\hline $941 \ldots \ldots \ldots \ldots \ldots \ldots \ldots$ & 1140 \\
\hline $946 \ldots \ldots \ldots \ldots \ldots \ldots$ & 1131 \\
\hline $947 \ldots \ldots \ldots \ldots \ldots \ldots$ & 1519 \\
\hline $952 \ldots \ldots \ldots \ldots \ldots \ldots \ldots$ & 1454 \\
\hline $965 \ldots \ldots \ldots \ldots \ldots \ldots \ldots$ & 1362 \\
\hline $968 \ldots \ldots \ldots \ldots \ldots \ldots \ldots$ & 1091 \\
\hline $970 \ldots \ldots \ldots \ldots \ldots \ldots \ldots$ & 991 \\
\hline $973 \ldots \ldots \ldots \ldots \ldots \ldots$ & 172 \\
\hline $978 \ldots \ldots \ldots \ldots \ldots \ldots \ldots$ & 1878 \\
\hline${ }^{\mathrm{c}} \ldots \ldots \ldots \ldots \ldots \ldots \ldots$ & 1141 \\
\hline $991 \ldots \ldots \ldots \ldots \ldots \ldots \ldots$ & 950 \\
\hline $992 \ldots \ldots \ldots \ldots \ldots \ldots \ldots$ & 727 \\
\hline $1007 \ldots \ldots \ldots \ldots \ldots \ldots \ldots$ & 1298 \\
\hline $1010 \ldots \ldots \ldots \ldots \ldots \ldots \ldots$ & 1507 \\
\hline $1015 \ldots \ldots \ldots \ldots \ldots \ldots$ & 1165 \\
\hline $1016 \ldots \ldots \ldots \ldots \ldots \ldots \ldots$ & 1375 \\
\hline $1019 \ldots \ldots \ldots \ldots \ldots \ldots \ldots$ & 650 \\
\hline $1023 \ldots \ldots \ldots \ldots \ldots \ldots \ldots$ & 1164 \\
\hline $1032 \ldots \ldots \ldots \ldots \ldots \ldots$ & 1684 \\
\hline $1034 \ldots \ldots \ldots \ldots \ldots \ldots \ldots$ & 1299 \\
\hline $1044 \ldots \ldots \ldots \ldots \ldots \ldots \ldots$ & 1947 \\
\hline $1049 \ldots \ldots \ldots$ & 1569 \\
\hline $1055 \ldots \ldots \ldots \ldots \ldots \ldots$ & 1543 \\
\hline $1060 \ldots \ldots$ & 1622 \\
\hline
\end{tabular}

TABLE 3-Continued

\begin{tabular}{|c|c|}
\hline $\mathrm{ID}^{\mathrm{a}}$ & $\begin{array}{c}v_{r}^{\mathrm{b}} \\
\left(\mathrm{km} \mathrm{s}^{-1}\right)\end{array}$ \\
\hline 1064. & 1405 \\
\hline$\ldots \ldots \ldots \ldots$ & 1100 \\
\hline 1067. & 1368 \\
\hline 1070 . & 1437 \\
\hline n........ & 1015 \\
\hline$\ldots \ldots \ldots \ldots$ & 905 \\
\hline $1101 \ldots$ & 1494 \\
\hline n........ & -31 \\
\hline n......... & 1930 \\
\hline$\ldots \ldots \ldots \ldots$ & 1091 \\
\hline $1113 \ldots$ & 1544 \\
\hline n......... & 1084 \\
\hline n......... & 1997 \\
\hline$\ldots \ldots \ldots \ldots$ & 1367 \\
\hline $1144 \ldots$ & 808 \\
\hline n........ & 1370 \\
\hline$\ldots \ldots \ldots \ldots \ldots$ & 1731 \\
\hline$\ldots \ldots \ldots \ldots$ & 1048 \\
\hline$\ldots \ldots \ldots \ldots$ & 1473 \\
\hline$\ldots \ldots \ldots \ldots$ & 1476 \\
\hline$\ldots \ldots \ldots \ldots \ldots \ldots$ & 1106 \\
\hline$\ldots \ldots \ldots \ldots \ldots$ & 639 \\
\hline 1200 . & 878 \\
\hline$\ldots \ldots \ldots \ldots$ & 1178 \\
\hline $1205 \ldots \ldots \ldots \ldots \ldots \ldots \ldots$ & 1011 \\
\hline n............ & 1605 \\
\hline n.......... & 101 \\
\hline$\ldots \ldots \ldots \ldots \ldots$ & 1125 \\
\hline n............. & 1244 \\
\hline$\ldots \ldots \ldots \ldots \ldots$ & 855 \\
\hline $1238 \ldots \ldots \ldots \ldots \ldots \ldots \ldots$ & 727 \\
\hline $1240 \ldots \ldots \ldots \ldots \ldots \ldots \ldots$ & 1359 \\
\hline $1244 \ldots \ldots \ldots \ldots \ldots \ldots \ldots$ & 1913 \\
\hline $1247 \ldots \ldots \ldots \ldots \ldots \ldots \ldots$ & 1672 \\
\hline$\ldots \ldots \ldots \ldots \ldots$ & 877 \\
\hline $1264 \ldots \ldots \ldots \ldots \ldots \ldots \ldots$ & 39 \\
\hline$\ldots \ldots \ldots \ldots \ldots \ldots$ & 0 \\
\hline $1290 \ldots \ldots \ldots \ldots \ldots \ldots \ldots$ & 728 \\
\hline $1291 \ldots \ldots \ldots \ldots \ldots \ldots \ldots$ & -57 \\
\hline $1293 \ldots \ldots \ldots \ldots \ldots \ldots \ldots$ & 776 \\
\hline $1298 \ldots \ldots \ldots \ldots \ldots \ldots \ldots$ & 195 \\
\hline $1301 \ldots \ldots \ldots \ldots \ldots \ldots \ldots$ & 1053 \\
\hline $1309 \ldots \ldots \ldots \ldots \ldots \ldots$ & 728 \\
\hline $1313 \ldots \ldots \ldots \ldots \ldots \ldots \ldots$ & 1264 \\
\hline $1322 \ldots \ldots \ldots \ldots \ldots \ldots \ldots$ & 1333 \\
\hline $1336 \ldots \ldots \ldots \ldots \ldots \ldots$ & 969 \\
\hline $1340 \ldots \ldots \ldots \ldots \ldots \ldots \ldots$ & 1296 \\
\hline $1341 \ldots \ldots \ldots \ldots \ldots \ldots \ldots$ & -41 \\
\hline $1344 \ldots \ldots \ldots \ldots \ldots \ldots \ldots$ & 982 \\
\hline $1346 \ldots \ldots \ldots \ldots \ldots \ldots \ldots$ & -178 \\
\hline $1351 \ldots \ldots \ldots \ldots \ldots \ldots \ldots$ & 1705 \\
\hline $1353 \ldots \ldots \ldots \ldots \ldots \ldots \ldots$ & 2161 \\
\hline $1356 \ldots \ldots \ldots \ldots \ldots \ldots$ & 3 \\
\hline $1367 \ldots \ldots \ldots \ldots \ldots \ldots \ldots$ & 1278 \\
\hline $1370 \ldots \ldots \ldots \ldots \ldots \ldots$ & 1041 \\
\hline $1382 \ldots \ldots \ldots \ldots \ldots \ldots \ldots$ & 1472 \\
\hline $1391 \ldots \ldots \ldots \ldots \ldots \ldots \ldots$ & 1186 \\
\hline $1400 \ldots \ldots \ldots \ldots \ldots \ldots \ldots$ & 915 \\
\hline $1409 \ldots \ldots \ldots \ldots \ldots \ldots$ & 1103 \\
\hline $1425 \ldots \ldots \ldots \ldots \ldots \ldots \ldots$ & 1338 \\
\hline $1431 \ldots \ldots \ldots \ldots \ldots \ldots \ldots$ & 1303 \\
\hline $1433 \ldots \ldots \ldots \ldots \ldots \ldots \ldots$ & 1917 \\
\hline $1449 \ldots \ldots \ldots \ldots \ldots \ldots \ldots$ & 1067 \\
\hline $1457 \ldots \ldots \ldots \ldots \ldots \ldots \ldots$ & 784 \\
\hline $1461 \ldots \ldots \ldots \ldots \ldots \ldots \ldots$ & 693 \\
\hline $1463 \ldots \ldots \ldots \ldots \ldots \ldots \ldots$ & 1871 \\
\hline $1469 \ldots \ldots$ & 1101 \\
\hline$\ldots \ldots \ldots \ldots \ldots$ & 447 \\
\hline $1481 \ldots \ldots \ldots \ldots \ldots \ldots \ldots$ & 1789 \\
\hline $1483 \ldots \ldots \ldots \ldots \ldots \ldots \ldots$ & 1625 \\
\hline $1490 \ldots \ldots \ldots \ldots \ldots \ldots \ldots$ & 1403 \\
\hline $1497 \ldots \ldots$ & 3 \\
\hline
\end{tabular}


TABLE 3-Continued

\begin{tabular}{|c|c|}
\hline $\mathrm{ID}^{\mathrm{a}}$ & $\begin{array}{c}v_{r}{ }^{\mathrm{b}} \\
\left(\mathrm{km}^{-1} \mathrm{~s}^{-1}\right)\end{array}$ \\
\hline $1504 \ldots$ & 626 \\
\hline $1514 \ldots$ & 1165 \\
\hline $1531 \ldots$ & 209 \\
\hline $1538 \ldots$ & 1237 \\
\hline $1540 \ldots$ & 587 \\
\hline $1548 \ldots$ & 1735 \\
\hline $1563 \ldots$ & 695 \\
\hline $1565 \ldots$ & 1574 \\
\hline $1577 \ldots$ & 1366 \\
\hline .............. & 1537 \\
\hline $1615 \ldots$ & 1168 \\
\hline $1617 \ldots$ & 1369 \\
\hline $1631 \ldots$ & 1157 \\
\hline …............ & 995 \\
\hline $1709 \ldots$ & 1790 \\
\hline
\end{tabular}

${ }^{a}$ Identification from Strom et al. 1981 except for added clusters near center of M87-see Table 1.

${ }^{\mathrm{b}}$ Heliocentric radial velocities.

${ }^{c}$ The spectrum of a second object 6"6 east of Strom 978 was found in the slitlet of Strom 978.

source depends on where the source is located within the width of the slit and on the seeing. For multislit spectra, the same effect operates. But now it depends on the accuracy of the astrometry and object positions used to design the slit mask and on the accuracy of alignment of the objects on the sky to the physical slit mask in the entrance aperture of the spectrograph. The individual errors may vary from object to object and may cause a spurious velocity dispersion.

We denote the quantity of interest, the one-dimensional difference between the location of the object and the center of its slitlet as seen by the spectrograph, both measured in the dispersion direction, as $\Delta(X)$. Detailed logs of each slit mask alignment procedure are available for most of the 1995 and 1996 LRIS observations. They indicate a median $1 \sigma \mathrm{rms}$ value for $\Delta(X)$ of 0 ". 4 . This corresponds to $2.3 \AA$ $\left(135 \mathrm{~km} \mathrm{~s}^{-1}\right.$ at $5100 \AA, 81 \mathrm{~km} \mathrm{~s}^{-1}$ at $\left.8500 \AA\right)$. Numerical simulations show that the actual errors introduced into the data are at least a factor of 2 smaller than this owing to the smoothing effects of the seeing.

These globular cluster spectra have much higher signalto-noise ratios than any previous M87 spectra. For a typical M87 globular cluster candidate with $B=21.5 \mathrm{mag}$, we accumulate, summed along the slit, $\approx 2220$ detected photoelectrons pixel $^{-1}$ in the dispersion direction in the skysubtracted spectra centered at $5500 \AA$ per $3000 \mathrm{~s}$ of exposure. The sky, avoiding strong emission lines, and in the outer parts of M87 is $<10 \%$ as bright. In many cases, the accuracy of the radial velocities is limited by the systematics of the definition of the wavelength scale rather than the signal-to-noise ratio of the spectra. This subject will be discussed in more detail in the second paper of this series on the abundances in the M87 globular clusters. Here we merely assert that the $1 \sigma$ uncertainty in the radial velocities of Table 3 is under $100 \mathrm{~km} \mathrm{~s}^{-1}$.

For high signal-to-noise ratio spectra, one can fairly easily achieve a centroiding error of 0.1 pixels, which translates directly into a $v_{r}$ error. Our adopted $v_{r}$ error of $100 \mathrm{~km}$ $\mathrm{s}^{-1}$ translates into 1.4 pixels ( 0.2 resolution elements) at
$5100 \AA$ and into 2.3 pixels ( 0.35 resolution elements) at 8500 $\AA$. Thus, our adopted radial velocity error of $100 \mathrm{~km} \mathrm{~s}^{-1}$ is indeed very conservative.

There are several ways to demonstrate that our data in fact achieve this level of accuracy. First, we note that the mean heliocentric radial velocity for the 205 M87 globular clusters in our sample is $1301 \pm 27 \mathrm{~km} \mathrm{~s}^{-1}$. The mean heliocentric $v_{r}$ for M87 itself based on long-slit high-dispersion spectroscopy of the nuclear region of the galaxy is $1277 \pm 5$ $\mathrm{km} \mathrm{s}^{-1}$ (van der Marel 1994). The agreement is extremely good.

Next we examine the objects in the final sample that have been observed more than once. Forty-two objects were observed twice, while one was observed 3 times. (Objects were observed twice because objects observed in the $\mathrm{Ca}$ triplet region were not automatically eliminated when designing masks to be used at $5500 \AA$. In addition, in certain places on the sky there were not many suitable objects, so the same candidate was selected to be included on multiple slit masks.) Figure 3 shows the histogram of the difference between the two measurements for the 43 objects that have been observed twice (including one observed 3 times). Table 4 gives the $\sigma$ for the velocity difference between pairs of

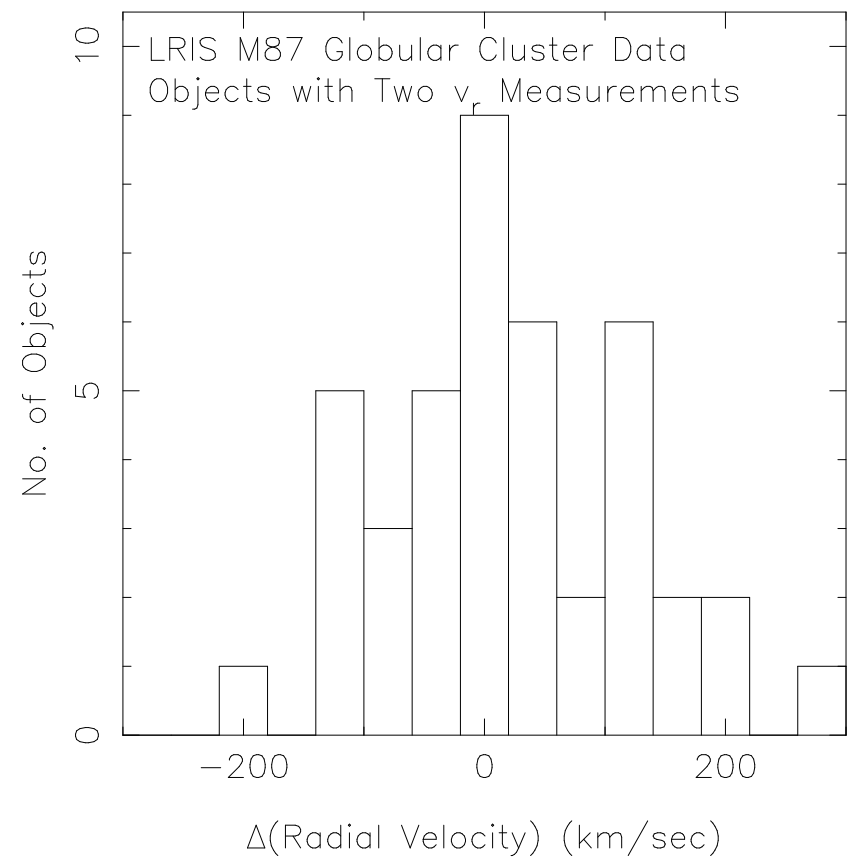

Fig. 3.- Histogram of the difference between the two independent $v_{r}$ determinations is shown for the 43 candidate globular clusters in M87 that have $v_{r}$ determined from more than one slit mask.

TABLE 4

$v_{r}$ COMPARISON FOR OBJECTS OBSERVED TWICE

\begin{tabular}{cccc}
\hline \hline $\begin{array}{c}\text { Spectral Range } \\
(\AA)\end{array}$ & $\begin{array}{c}\text { Number of } \\
\text { Objects }\end{array}$ & $\begin{array}{c}\sigma[\Delta(v)] \\
\left(\mathrm{km} \mathrm{s}^{-1}\right)\end{array}$ & $\begin{array}{c}\text { Inferred } \sigma\left(v_{r}\right) \\
\left(\mathrm{km} \mathrm{s}^{-1}\right)\end{array}$ \\
\hline Any $\ldots \ldots \ldots \ldots$. & 43 & 117 & 83 \\
$5200-5200 \ldots \ldots$. & 17 & 134 & 95 \\
$5200-8500 \ldots \ldots$. & 23 & 80 & $68^{\mathrm{b}}$ \\
$8500-8500 \ldots \ldots$ & 3 & 60 & 42 \\
\hline
\end{tabular}

${ }^{\text {a }}$ Central wavelength of multislit spectrum.

b This applies to a spectrum at $5200 \AA$ assuming the results of the last row of this table. 
measurements where both spectra are in the 8500 A region, pairs with one spectrum at the infrared $\mathrm{Ca}$ triplet and the second at the Mg triplet, and pairs with both spectra centered at the $\mathrm{Mg}$ triplet. The final column of Table 4 gives the $\sigma$ for a single radial velocity measurement inferred from the $\sigma$ of the differences of pairs of measurements. Table 4 confirms, as expected, that the accuracy of $v_{r}$ from the spectra centered at $8500 \AA$ is higher than that from the spectra centered at $5100 \AA$. Again, we conclude that $100 \mathrm{~km} \mathrm{~s}^{-1}$ is a conservative value for the $1 \sigma$ uncertainties in our radial velocities of candidate globular clusters in M87.

\section{ROTATION}

The isophotes of M87 are not circular. Many analyses of surface photometry for this galaxy exist (see, for example, Cohen 1986), and they have established that the ellipticity increases outward, reaching about $10 \%$ at a semimajor axis of about $80^{\prime \prime}$, with a major axis position angle of $155^{\circ} \pm 5^{\circ}$.

There is no evidence for rotation exceeding $20 \mathrm{~km} \mathrm{~s}^{-1}$ in amplitude in M87 from optical spectroscopy. However, the available data are often restricted to a single position angle, usually the minor axis, and to a small slit length on the sky (an effort to obtain maximum spatial resolution near the nucleus to look for a nuclear black hole). Such is the case for the kinematic studies of Bender, Saglia, \& Gerhard (1994) and van der Marel (1994). Jarvis \& Peletier (1991) looked at four position angles with a slit that covered $\pm 25^{\prime \prime}$ from the nucleus and found a maximum amplitude of rotation of 20 $\mathrm{km} \mathrm{s}^{-1}$. The recent work of Sembach \& Tonry (1996) reaches to about $100^{\prime \prime}$ out from the nucleus of M87. Although the authors say nothing about rotation, based on their long-slit spectrum along the major axis, there is marginal evidence for it with an amplitude of not more than 30 $\mathrm{km} \mathrm{s}^{-1}$ and with the southeast sector of the galaxy having the larger radial velocity than the northwest sector.

We solve for rotation in the globular cluster system of M87 using the Levenberg-Marquardt nonlinear leastsquares fitting routines from Press et al. (1986) to fit a sinusoid in position angle (plus a constant mean velocity) to the observed radial velocities of the 205 globular clusters. The results for all the clusters, and then for them divided into two radial bins, are given in Table 5 , where the number of M87 globular clusters in the sample, the mean $v_{r}$, the rotational velocity $\left(V_{\text {rot }}\right)$, and position angle at which the rotational contribution to $v_{r}$ is largest are given. The uncertainty in $V_{\text {rot }}$ is about $20 \%$.

Figure 4 shows the rotation curve as a function of position angle for the 205 globular clusters over two cycles. The solid line indicates the fit to the sample as a whole given on the first line of Table 5. This figure does not appear as convincing as might be desired. So we carried out simulations where the velocities of the M87 globular clusters were kept at their observed values, but their position angles were replaced by random values between $0^{\circ}$ and $360^{\circ}$, and the

TABLE 5

Solution for Rotation in the M87 Globular Cluster System

\begin{tabular}{crccc}
\hline $\begin{array}{c}\text { Radial Range } \\
(\operatorname{arcsec})\end{array}$ & $N_{\text {obs }}$ & $\begin{array}{c}\left\langle v_{r}\right\rangle \\
\left(\mathrm{km} \mathrm{s}^{-1}\right)\end{array}$ & $\begin{array}{c}V_{\text {rot }} \\
\left(\mathrm{km} \mathrm{s}^{-1}\right)\end{array}$ & $\begin{array}{c}\text { Position Angle } \\
(\mathrm{deg})\end{array}$ \\
\hline All $\ldots \ldots \ldots \ldots$ & 205 & 1298 & 100 & 145 \\
$R<180 \ldots \ldots$. & 79 & 1331 & 112 & 162 \\
$R>180 \ldots \ldots$ & 126 & 1275 & 95 & 134 \\
\hline
\end{tabular}

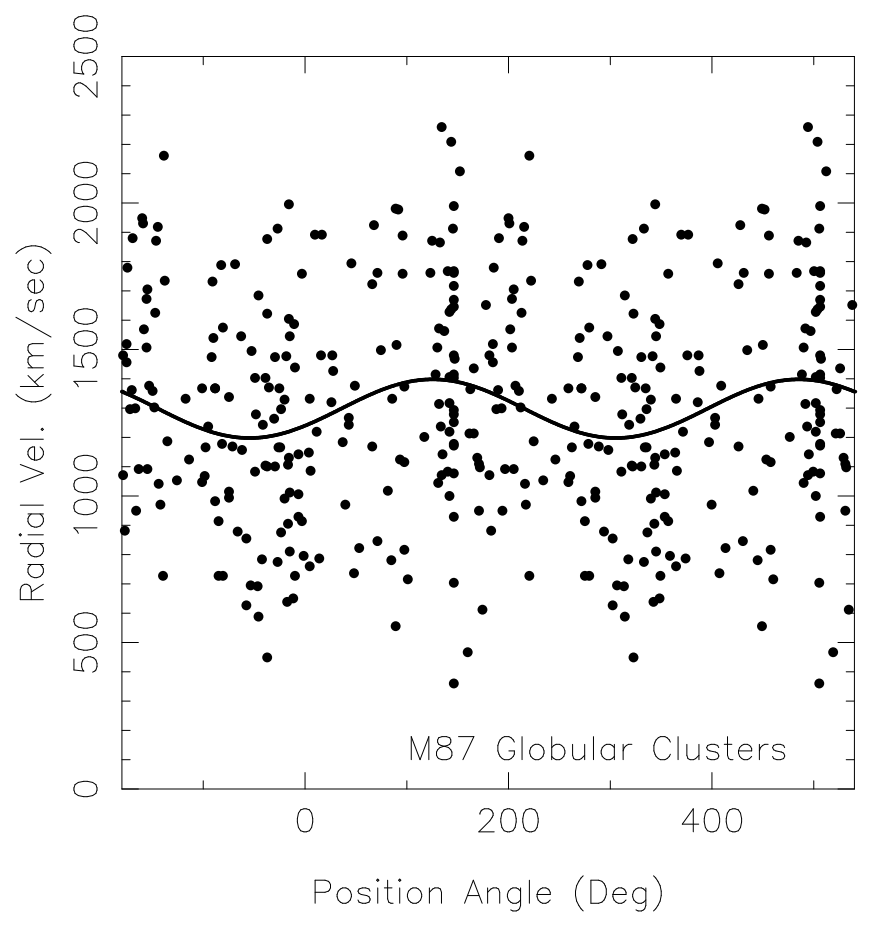

FIG. 4.-Solution for the projected rotation of M87 as inferred from the entire sample is shown as the solid curve. The velocities of the individual globular clusters in M87 are indicated as filled points.

data set was then analyzed for rotation. We conducted 200 trials of this procedure. These trials indicate that only $4 \%$ of the time does the artificial data show rotation as large as or larger than that of the actual data for the M87 globular clusters presented here.

Thus we infer a definite rotation in M87 that is approximately (to within $30^{\circ}$ ) about the minor axis of the galaxy as defined from its intensity isophotes. $V_{\text {rot }}$ is about $100 \mathrm{~km}$ $\mathrm{s}^{-1}$. This study, Sembach \& Tonry's (1996) long-slit spectroscopy, and Mould et al.'s (1990) earlier analysis of the M87 globular cluster system all agree on the direction of the rotation (larger $v_{r}$ along the major axis to the southeast, smaller $v_{r}$ to the northwest).

We need to adopt a definite rotational velocity profile in order to correct the observed radial velocities. For this purpose, we adopt a truncated solid body law, $V_{\text {rot }}=0.80 R$ $\cos \left(\theta-\mathrm{PA}_{0}\right)$ with the restriction that $V_{\text {rot }} \leq 180 \mathrm{~km} \mathrm{~s}^{-1}$, where $R$ is the projected radius (in arcsec) and $\mathrm{PA}_{0}=140^{\circ}$. The exact nature of the form adopted for $V_{\text {rot }}$ is not important, since in the next section we will show that rotation is small compared to the intrinsic velocity dispersion of the M87 globular cluster system.

We have carried out a Kolmogorov-Smirnov test on the observed radial velocity histogram of the M87 globular cluster system with the rotational corrections included. We find that more than $90 \%$ of the samples of 205 points drawn from a normal distribution with a mean and variance matching that of our ensemble will differ more from a Gaussian than do the actual data for the M87 globular clusters presented here.

\section{VELOCITY DISPERSIONS}

We are now in a position to compute the velocity dispersions. The 205 objects believed to be bona fide globular clusters in M87 are included. Heliocentric corrections are 
applied, and the rotation is removed using the prescription given above.

The M87 globular cluster sample is divided into a number of radial bins, and the mean velocity and velocity dispersion are calculated for each bin. Then, the most discrepant object is eliminated, and the mean and dispersion are calculated again. Table 6 gives the results with and without the rotational correction described above. The differences between the two are not large. The sampling uncertainties in $\sigma\left(v_{r}\right)$ assuming $v_{r}$ has a Gaussian distribution of $\sigma\left(v_{r}\right) /\left(2 N_{\mathrm{bin}}\right)^{1 / 2}$ are also given for one case.

In the final column of Table 6 , we give the values to be adopted for subsequent use. They include the rotation correction, and an instrumental uncertainty of $100 \mathrm{~km} \mathrm{~s}^{-1}$ has been removed in quadrature.

The velocity dispersion for the early-type galaxies in the Virgo Cluster is $570 \mathrm{~km} \mathrm{~s}^{-1}$ (Binggeli, Tammann, \& Sandage 1987), even larger than that of our outermost point.

\section{SIMPLE MASS MODELS}

Our goal is to establish in a definitive manner whether or not dark matter exists in the halo of M87, how much dark matter exists, and how certain we are of such claims.

We wish to find a distribution of mass within M87 that reproduces the velocity dispersion as a function of projected radius $(R)$ that we have derived from our observations of the M87 globular cluster system. There are many observations of the surface brightness of M87, so once a mass distribution is available, the mass-to-light ratio can easily be computed. "Light" throughout this discussion refers to the integrated light at $V(5500 \AA)$ of M87, which is dominated by the stellar component of M87, although the M87 globular clusters do make a small contribution as well.

As a first reconnaissance, although we know that the outer isophotes of M87 are not circular, the ellipticity is small, and we treat $\rho(r)$ as spherical, where $r$ is the radius. We begin by assuming that the mass is distributed as is the light of M87, i.e., $M / L$ is constant.

Hernquist (1990) proposed a general model density distribution for spherical galaxies that has some very desirable properties. Over a wide range in radius, the projected mass derived from this density distribution matches the wellknown $R^{0.25}$ dependence for surface brightness. Many properties of this density distribution, including the line-ofsight velocity dispersion for both isotropic and circular orbits, can be expressed as analytical functions. This model, which is the model used by Mould et al. (1990), has two free parameters, a spatial scale $(A)$ and a total mass, $M$.

Since a Hernquist model with $A=52^{\prime \prime}(3.8 \mathrm{kpc})$ fits the M87 surface brightness profile, we proceed to use this analytical mass model. We use the surface brightness measurements of Boroson, Thompson, \& Shectman (1983) and of de Vaucouleurs \& Nieto (1978) to integrate numerically for the enclosed surface brightness $[L(R)]$ and use the model only to calculate the deprojection factor $L(r) / L(R)$. We assume a galactic absorption $A_{V}$ of $0.14 \mathrm{mag}$. We assume a distance of M87 of $15 \mathrm{Mpc}$ (corresponding to a scale on the sky of 73 pc $\operatorname{arcsec}^{-1}$ ) based on the Cepheid results for spirals in the Virgo Cluster (Saha et al. 1995; Pierce et al. 1994; Ferrarese et al. 1996).

We assume an isotropic system $\left[\sigma^{2}\left(v_{r}\right)=\sigma^{2}\left(v_{\theta}\right)=\sigma^{2}\left(v_{\phi}\right)\right]$. We then adjust the only remaining free parameter of the Hernquist (1990) model, the total mass $M$, so that the lineof-sight velocity dispersion matches the observed values for the first three radial bins. The required value of $M$ is $7.5 \times 10^{11} M_{\odot}$. The enclosed $M(r)$ relationship for this model, if represented as a power law, has an exponent of 0.5 over the region of interest. Equation (41) of Hernquist (1990) is the analytical solution for the projected velocity dispersion for isotropic orbits, which we use to calculate the projected velocity dispersion we predict for the stars in M87.

The probes we are using, i.e., the M87 globular clusters, have a more extended areal distribution, $n_{\mathrm{GC}}$, than does the M87 stellar halo light (Harris 1986; Cohen 1986; McLaughlin, Harris, \& Hanes 1994). $n_{\mathrm{GC}}$ also has an approximately constant surface density in a core $60^{\prime \prime}$ in radius (more than 10 times larger than that of M87 itself) (Lauer \& Kormendy 1986). We adopt the analytical function fit to $n_{\mathrm{GC}}$ of Merritt \& Tremblay (1993). The Abel projection integral is then numerically inverted to obtain the volume density of globular clusters, $\rho_{\mathrm{GC}}$.

To calculate the projected velocity distribution expected for the M87 globular clusters, the Jeans equation in spherical coordinates (eq. [4-55] of Binney \& Tremaine 1994) was integrated numerically using the Hernquist potential with the values for the parameters given above.

The solid curve in Figure 5 shows the predicted line-ofsight velocity dispersion as a function of projected radius. The thick solid line denotes that expected for the M87 globular clusters, while the thin solid line denotes that

TABLE 6

Velocity Dispersions for the M87 Globular Cluster System

\begin{tabular}{|c|c|c|c|c|c|c|c|c|}
\hline $\begin{array}{l}\text { Radial Range } \\
\quad(\operatorname{arcsec})\end{array}$ & $\begin{array}{l}\text { Number of } \\
\text { Clusters }\end{array}$ & $\begin{array}{c}\left\langle v_{r}\right\rangle \\
\left(\mathrm{km} \mathrm{s}^{-1}\right)\end{array}$ & $\begin{array}{c}\sigma\left(v_{r}\right)(N) \\
\left(\mathrm{km} \mathrm{s}^{-1}\right)\end{array}$ & $\begin{array}{c}\sigma\left(v_{r}\right)(N-1)^{\mathrm{a}} \\
\left(\mathrm{km} \mathrm{s}^{-1}\right)\end{array}$ & $\begin{array}{c}\left\langle v_{r}\right\rangle^{\mathbf{b}} \\
\text { (derot) }\end{array}$ & $\begin{array}{c}\sigma\left(v_{r}\right)(N)(\text { derot }) \\
\left(\mathrm{km} \mathrm{s}^{-1}\right)\end{array}$ & $\begin{array}{c}\sigma\left(v_{r}\right)(N-1)^{\mathrm{a}} \\
(\mathrm{derot}) \\
\left(\mathrm{km} \mathrm{s}^{-1}\right)\end{array}$ & $\begin{array}{c}\sigma\left(v_{r}\right)(N-1)^{\mathrm{c}} \\
(\text { derot, corr) } \\
\left(\mathrm{km} \mathrm{s}^{-1}\right)\end{array}$ \\
\hline$<70$ & 19 & 1435 & 310 & 271 & 1410 & 318 & $279(66)^{d}$ & 260 \\
\hline $70<R<103 \ldots \ldots$ & 24 & 1410 & 335 & 288 & 1384 & 344 & $291(61)$ & 273 \\
\hline $105<R<146 \ldots \ldots$ & 24 & 1220 & 290 & 273 & 1208 & 272 & $243(51)$ & 221 \\
\hline $146<R<197 \ldots \ldots$ & 24 & 1350 & 440 & 407 & 1337 & 411 & 360 (75) & 346 \\
\hline $198<R<241 \ldots \ldots$ & 24 & 1235 & 327 & 298 & 1245 & 341 & $294(61)$ & 276 \\
\hline $244<R<295 \ldots \ldots$ & 24 & 1176 & 417 & 368 & 1156 & 483 & $425(89)$ & 413 \\
\hline $296<R<328 \ldots \ldots$ & 24 & 1271 & 366 & 339 & 1277 & 390 & $352(73)$ & 337 \\
\hline $330<R<382 \ldots \ldots$ & 24 & 1230 & 379 & 357 & 1275 & 381 & $348(73)$ & 333 \\
\hline $386<R<517 \ldots \ldots$ & 18 & 1443 & 556 & 500 & 1474 & 571 & $486(118)$ & 476 \\
\hline
\end{tabular}

a $\sigma\left(v_{r}\right)$ calculated with the most discrepant cluster omitted.

${ }^{b}$ The rotation of the M87 globular cluster system has been removed.

${ }^{c}$ A correction for an instrumental uncertainty of $100 \mathrm{~km} \mathrm{~s}^{-1}$ has been removed in quadrature.

${ }^{\mathrm{d}}$ The values in parentheses are the $1 \sigma$ sampling uncertainties for these dispersions. 


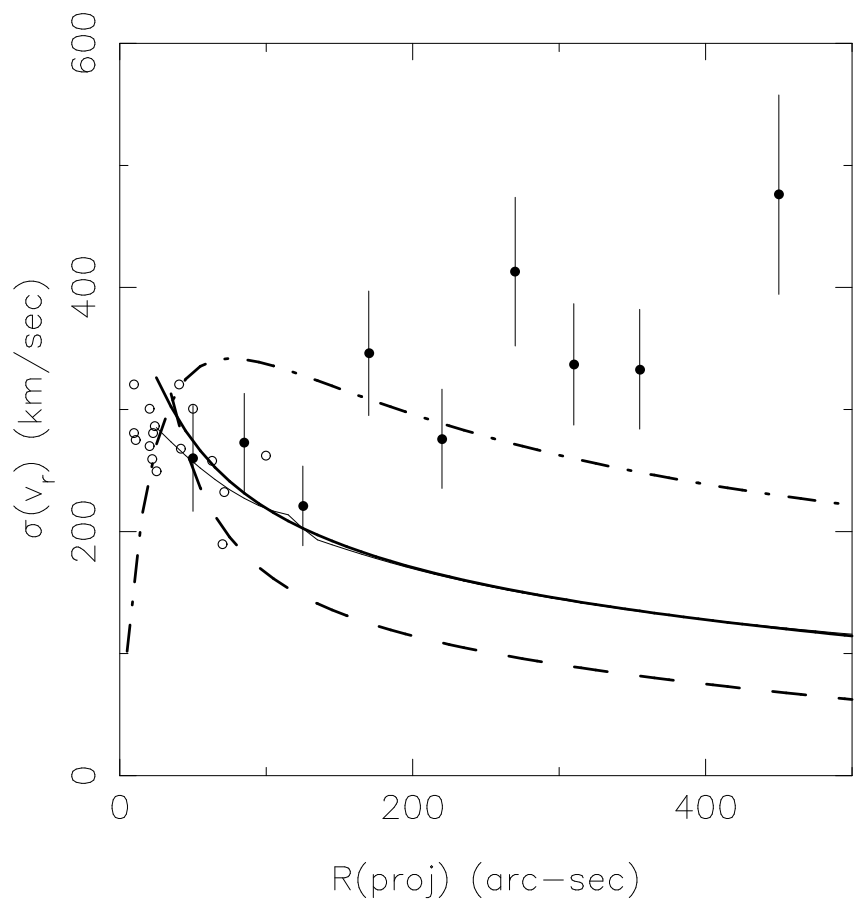

Fig. 5.-Line-of-sight velocity dispersion $\sigma\left(v_{r}\right)$ for the M87 globular cluster sample is shown as a function of projected radius. The open circles denote optical measurements of $\sigma\left(v_{r}\right)$ for the stellar component of M87. Assuming constant $M / L$, the thin curve represents $\sigma\left(v_{r}\right)(R)$ predicted for the stellar halo of M87. The thick curves represent $\sigma\left(v_{r}\right)(R)$ predicted for the globular cluster system of M87 with isotropic orbits yielding the solid line, radial orbits yielding the dashed line, and circular orbits yielding the dotdashed line.

expected for the stellar halo of M87. The filled circles in the figure denote the values deduced from our measurements of the M87 globular cluster system taken from the final column of Table 6 . The results from optical long-slit spectroscopy for $R \geq 20^{\prime \prime}$, half of which are from Sargent et al. (1978) and the remainder from Bender et al. (1994), van der Marel (1994), and Sembach \& Tonry (1996), are indicated by the open circles. (The two values adopted from Sembach \& Tonry 1996 have been corrected downward by $7 \%$ as suggested in their paper.) The agreement between the optical long-slit spectroscopy and our data is good.

TABLE 7

M87 M/L From A ToY ModeL FOR THE MASS OF THE M87 Globular Cluster System ${ }^{\mathrm{a}}$

\begin{tabular}{cc}
\hline \hline $\begin{array}{c}R \\
(\mathrm{kpc})\end{array}$ & $\begin{array}{c}M / L^{\mathrm{b}} \\
(\text { solar })\end{array}$ \\
\hline $3.6 \ldots \ldots$. & 12 \\
$6.9 \ldots \ldots$. & 16 \\
$9.1 \ldots \ldots$. & 12 \\
$12.7 \ldots \ldots$. & 21 \\
$16.0 \ldots \ldots$. & 23 \\
$18.9 \ldots \ldots$. & 58 \\
$21.9 \ldots \ldots$. & 47 \\
$25.9 \ldots \ldots$. & 55 \\
$32.8 \ldots \ldots$. & 134 \\
\hline
\end{tabular}

${ }^{\text {a }}$ A central point mass is used.

b "Light" is the integrated light of the stellar component of M87 at $5500 \AA$.
Figure 5 shows clearly how tightly confined toward the nucleus the optical data is, which is an indication of the intense interest in the search for a nuclear black hole as well as the fact that the rapidly decreasing surface brightness of the M87 stellar halo makes such observations at larger radii impossible.

The model with mass following the light of M87, which by definition has a constant mass-to-light ratio, does not fit the data.

In an effort to find a suitable fit, we try varying the orbital distribution of the M87 globular clusters. The dot-dashed line in Figure 5 represents the predicted line-of-sight velocity dispersion for the M87 globular cluster system assuming purely circular orbits in the Hernquist potential whose parameters ( $M$ and $A$ ) have the values adopted above. The dashed line represents a system under these conditions with radial orbits. This curve was obtained by numerical integration of the spherical Jeans equation with the appropriate orbital anisotropy term. One might adopt an orbital distribution for the M87 globular clusters that varies with $r$, depending on one's prejudices about globular cluster formation and survival. For example, clusters with radial orbits might not survive at small $r$ but might survive at large $r$ where they do not undergo repeated passages through the nucleus of M87. However, one expects a system with a fixed total mass and with an intermediate family of orbits to lie within the area bounded by the upper and lower of the three thick curves in Figure 5. The range of behavior shown by the various orbital distributions is not capable of explaining the observed data within the assumption of a fixed $M / L$ ratio.

The distribution of $v_{r}$ of the M87 globular clusters provides an additional constraint on the orbital characteristics although it probably requires a larger sample of M87 globular clusters to exploit this (Merritt 1997; Merritt, Meylan, \& Mayor 1997).

To get some idea of what kind of a mass model is in fact required to match the data, we adopt as a toy model a central point mass and isotropic orbits for the probes. The central mass required to match the data in each radial bin, $M(r)$, can then be calculated directly from $\sigma_{v}^{2}=G M /(4 r)$ (eq. [10-6] of Binney \& Tremaine 1994), where $r$ is a radius, not a projected radius. Assuming galaxies are located at their projected radii, we calculate $\left\langle\sigma_{v}^{2}\right\rangle\langle r\rangle$ for each radial bin to obtain $M(r)$. This yields the mass-to-light ratios as a function of $r$ given in Table 7, with $M \sim r^{1.5}$. The required $M / L$ values in the outer part of M87 are very large, more than 10 time those of the inner part of M87.

Changing the orbital characteristics for the M87 globular clusters (isotropic versus circular vs. radial orbits) and allowing for the corrections associated with a proper calculation of the gravitating mass will not alter the fundamental result that there must be a substantial amount of dark matter in the outer part of M87, that $M \sim R^{\alpha}$ with $\alpha>1$.

\section{FINAL MASS MODEL}

To derive a mass model, we solve the spherical Jeans equation assuming isotropic orbits and spherical symmetry

$$
M(r)=-\left[\frac{r \sigma(v)^{2}}{G}\right] \times\left[\frac{d \ln \left(\rho_{\mathrm{GC}}\right)}{d \ln (r)}+\frac{d \ln \sigma(v)^{2}}{d \ln (r)}\right] .
$$

To evaluate the first term on the right-hand side, we use $\rho_{\mathrm{GC}}(r)$ calculated earlier. The logarithmic derivative here 
TABLE 8

M87 MASS MODEL INFERRED FROM ITS Globular Cluster System

\begin{tabular}{ccc}
\hline \hline $\begin{array}{c}r \\
(\mathrm{kpc})\end{array}$ & $\begin{array}{c}M(r) \\
(\text { solar})\end{array}$ & $\begin{array}{c}\langle M / L\rangle^{\mathrm{a}} \\
(\text { solar })\end{array}$ \\
\hline $3.6 \ldots \ldots$. & $0.7( \pm 0.3) \times 10^{11}$ & $4.2^{\mathrm{b}}$ \\
$6.2 \ldots \ldots$. & $2.0( \pm 0.6) \times 10^{11}$ & 8.1 \\
$9.1 \ldots \ldots$. & $2.4( \pm 0.6) \times 10^{11}$ & 6.6 \\
$12.4 \ldots \ldots$ & $8.1( \pm 2.3) \times 10^{11}$ & 18.8 \\
$16.1 \ldots \ldots$. & $6.6( \pm 2.1) \times 10^{11}$ & 14.4 \\
$19.7 \ldots \ldots$ & $1.8( \pm 0.5) \times 10^{12}$ & 38.6 \\
$22.6 \ldots \ldots$ & $1.4( \pm 0.4) \times 10^{12}$ & 27.2 \\
$25.9 \ldots \ldots$ & $1.4( \pm 0.4) \times 10^{12}$ & 28.2 \\
$32.9 \ldots \ldots$ & $3.8( \pm 1.2) \times 10^{12}$ & 74.4 \\
\hline
\end{tabular}

a $\langle M / L\rangle$ is the mean value inside a sphere of radius $r$.

b "Light" is the integrated light of the stellar component of M87 at $5500 \AA$.

varies from -1.4 within the flat core of the globular cluster distribution in the inner part of M87 to -3.6 for the outer part. We deproject the observed velocity dispersions by fitting $\sigma(v)$ as a function of $R$ with a linear fit $[\sigma(v)(\mathrm{km}$ $\left.\left.\mathrm{s}^{-1}\right)=216+0.49 R(\operatorname{arcsec})\right]$. [The deprojection term for $\sigma(v)$ is not large, never exceeding the $15 \%$, and numerical integrations using $\rho_{\mathrm{GC}}(r)$ give very similar results.] This allows us to evaluate the logarithmic derivative term analytically-it varies from $\approx 0.2$ in the inner part of M87 to about 0.8 for the outer parts.

For our outermost point, $\left[-d \ln \left(\rho_{\mathrm{GC}}\right) / d \ln (r)\right]$ $-\left[d \ln \sigma(v)^{2} / d \ln (r)\right]$ is 2.7 , while the toy model described above has a factor of 4 . This indicates the magnitude of the correction factors from a simple central point mass spherical model with isotropic velocities and provides some guidance as to the magnitude of the remaining model-dependent uncertainties, which are primarily the choice of the orbital characteristics for the M87 globular cluster system.

The results are given in Table 8 . The uncertainties indicated for $M(r)$ are from sampling errors only and do not include any contribution for modeling uncertainties, problems with the rotation corrections, etc. Also note that the $\langle M / L\rangle$ at $r$ is the ratio averaged over the enclosed volume, not the value at $r$ itself.

Sargent et al. (1978) obtained $\langle M / L\rangle \approx 7$ (using the same definition of "light" as we are using) between $r=50$ and 71".8, their outermost point, a result reproduced by Saglia, Bertin, \& Stiavelli (1992) with their more sophisticated models. The agreement for the region of overlap of the two data sets is good.

Mould et al. (1990), with a very limited sample of data, found $M(39 \mathrm{kpc})=2.4 \times 10^{12} M_{\odot}$, in reasonable agreement with the above. Our much larger sample has allowed us to put their preliminary results on a much firmer basis and to derive a detailed model for the behavior of $M(r)$.

Fitting a power law to $M(r)$ yields an exponent of 1.7 , and the $M / L$ ratios must be very large $(\geq 30)$ in the outer part of M87. There is no way of reworking the data, the assumptions, or the analysis that avoids this conclusion.

\section{THE X-RAY VIEW OF M87}

Very extended X-ray emission centered on M87 has been detected and can also be used to infer the gravitating mass of M87. Here, one assumes hydrostatic equilibrium and that the velocity distribution of the gas particles is Maxwellian (isotropic) with a velocity dispersion related to the temperature $T_{\mathrm{X}}$ of the X-ray-emitting gas in the usual way. The surface brightness of the X-ray emission can be measured by an X-ray-imaging camera with suitable sensitivity, and reasonably precise measurements were obtained by the Einstein satellite. The problem is the determination of $T_{\mathrm{X}}-$ such measurements were beyond the state of the art until quite recently.

Fabricant \& Gorenstein (1983) analyzed the Einstein data for M87. They concluded that $M / L$ increased from $\approx 10$ at $r=1^{\prime}$ to over 180 at $r=20^{\prime}(\approx 87 \mathrm{kpc})$. They found a total mass of M87 within $20^{\prime}$ of M87 of $1.2-1.9 \times 10^{13} M_{\odot}$. Though they could follow the surface brightness in a broad X-ray bandpass out to $90^{\prime}$, they could not determine $T_{\mathrm{X}}$ beyond $25^{\prime}$. The uncertainty in their determinations of $T_{\mathrm{X}}(r)$ was large, and they quote results for many models of this parameter. The values given in Table 9 are for their model 1, an isothermal model with $T_{\mathrm{X}}=3 \mathrm{keV}$. Tsai $(1993,1994)$ has tried a more complex multiphase model of the Einstein data. He obtains similar results.

Nulson \& Böhringer (1995) provide a determination of the mass of the central Virgo Cluster using ROSAT data. Their determinations for $M(r)$ in the inner $100 \mathrm{kpc}$ centered on M87 are about 2.5 times smaller than those of Fabricant $\&$ Gorenstein (1983), partly owing to their use of a lower $T_{\mathbf{X}}$ there. Meanwhile, $A S C A$, with its superb spectral resolution in the X-ray regime, has observed M87 as well (Matsumoto et al. 1996). The $A S C A$ data convincingly demonstrate that at least two components are required to fit the X-ray spectra, with $T_{\mathrm{X}}$ of 3 and $1.3 \mathrm{keV}$, respectively. The hotter component has a more extended spatial distribution, and for both components, $T_{\mathrm{X}}$ is approximately constant with $r$. It is not obvious how to correct the ROSAT M(r) determinations in light of these new $A S C A$ results, as the ratio of the emission measure for the hot and cool components of the gas is changing rapidly over the spatial region of interest.

The best power-law fit to the ROSAT results of Nulsen \& Böhringer (1995) in the regime $50<r<100 \mathrm{kpc}$ is $M \sim r^{1.4}$, close to the $M \sim r^{1.7}$ found from the M87 globular cluster system.

$M(r)$ for the outer part of M87 is displayed in Figure 6. The solid points indicate the results from the present globular cluster data. The $1 \sigma$ uncertainties shown include only observational limitations and do not include the modeling uncertainties, which are at most $50 \%$. The best-fit power

TABLE 9

The Mass OF M87 FROM X-RAY MisSIONS AND FROM Its Globular Cluster System

\begin{tabular}{cccc}
\hline \hline $\begin{array}{c}r \\
(\mathrm{kpc})\end{array}$ & $\begin{array}{c}M(r)^{\mathrm{a}} \\
(\text { solar })\end{array}$ & $\begin{array}{c}M(r)^{\mathrm{b}} \\
(\text { solar })\end{array}$ & $\begin{array}{c}M(r)^{\mathrm{c}} \\
(\text { solar })\end{array}$ \\
\hline Gas: & & & \\
$44 \ldots \ldots$. & $6.2 \times 10^{10}$ & $7.5 \times 10^{10}$ & \\
$88 \ldots \ldots$. & $2.1 \times 10^{11}$ & $3.0 \times 10^{11}$ & \\
& & & \\
Total Mass: & & & \\
$44 \ldots \ldots$. & $6.4 \times 10^{12}$ & $1.5 \times 10^{12}$ & $3.0 \times 10^{12}$ \\
$88 \ldots \ldots$. & $1.3 \times 10^{13}$ & $5.0 \times 10^{12}$ & \\
\hline
\end{tabular}

\footnotetext{
${ }^{a}$ Einstein data analyzed by Fabricant \& Gorenstein 1983.

${ }^{\mathrm{b}}$ ROSAT data analyzed by Nulsen \& Böhringer 1995.

${ }^{c}$ Our analysis of the dynamics of the M87 globular cluster system.
} 


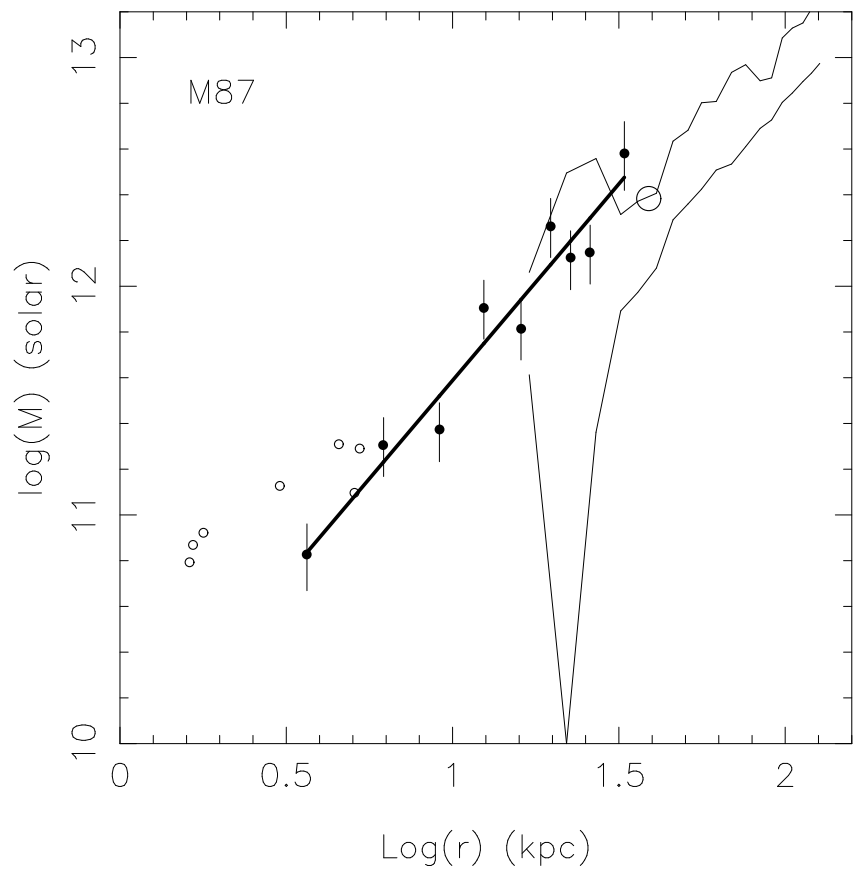

Fig. 6.-Filled circles denote $M(r)$ as derived here from the globular cluster system of M87. The upper and lower limits on $M(r)$ from the ROSAT data of Nulsen \& Böhringer (1995) are shown as the thin solid lines. The large open circle is the value of Mould et al. (1990). The small open circles are from Sargent et al. (1978). The best-fit power law to the globular cluster data is shown as a solid line.

law to these data is shown as the thick solid line. The large open circle is the result of Mould et al. (1990), while the X-ray upper and lower limits from Nulson \& Böhringer (1995) are shown as thin solid lines. The small open circles denote the values of Sargent et al. (1978) from optical spectroscopy of the stellar component of M87. The overall agreement among these various methods, which rely on different sets of physical assumptions and measure the gravitating mass using different components of M87 as probes, in the regions of overlap is very gratifying.

\section{SUMMARY}

This paper presents an analysis of the dynamics of the M87 globular cluster system with the goal of determining the $M / L$ ratio in the outer parts of this massive galaxy in the core of the Virgo Cluster. A sample of globular cluster candidates in M87 has been observed using slit masks with the LRIS on the Keck I 10 m telescope. After eliminating fore- ground galactic stars and background galaxies, we isolated 205 bona fide members of M87 globular cluster system.

These globular clusters belong to M87; their mean $v_{r}$ is that of M87 itself to within the (very small) observational error. Their velocity distribution is consistent with that of a Gaussian about the mean $v_{r}$. There is also evidence for rotation of the globular cluster system of M87.

The observed velocity dispersion of the M87 globular clusters increases with projected radius from about $270 \mathrm{~km}$ $\mathrm{s}^{-1}$ at $r=9 \mathrm{kpc}$ to $\approx 400 \mathrm{~km} \mathrm{~s}^{-1}$ at $r=40 \mathrm{kpc}$. The inferred $M / L$ increases from 5 for $r<9 \mathrm{kpc}$ to $\approx 30$ for $r \approx 30 \mathrm{kpc}$, where "light" refers to the integrated light of the stellar component of M87 at 5500 A. $M(44$ $\mathrm{kpc})=3 \times 10^{12} M_{\odot}$, with $M \sim r^{1.7}$ for $4<R<33 \mathrm{kpc}$. No permitted changes in the data analysis, assumptions, or model can eliminate the need for a substantial extended halo of dark matter in M87.

These results fit reasonably well with those of optical long-slit spectroscopy at the innermost radii and onto those from X-ray satellites at the larger radii.

In spiral galaxies, determination of the properties of the dark matter halo from rotation curves is complicated by the presence of multiple components (disk, bulge, and halo) (Kent 1987), and the properties of at least the first two of these are strongly variable from galaxy to galaxy, as well as by data that in most cases do not reach out as far in radius. In M87, however, the dark halo dominates at $r \geq 10 \mathrm{kpc}$, and we have been able to infer its spatial distribution in some detail. However, M87 can hardly be described as a normal elliptical galaxy since it is located at the center of a massive cluster of galaxies surrounded by a very extensive halo of hot gas. An obvious task for the future is to look for evidence of dark matter in and around normal elliptical galaxies.

A discussion of the implications of these results for the formation of the globular cluster system of M87 will be presented in the second paper in this series with the abundance analyses.

The entire Keck/LRIS user community owes a huge debt to Jerry Nelson, Gerry Smith, Bev Oke, and many other people who have worked to make the Keck Telescope and LRIS a reality. We are grateful to the W. M. Keck Foundation, and particularly its late president, Howard Keck, for the vision to fund the construction of the W. M. Keck Observatory. We thank the referee, Tad Pryor, for his constructive suggestions that improved the analysis presented here. Publication charges were paid by NSF grant AST9616729.
Bender, R., Saglia, R. R., \& Gerhard, O. E. 1994, MNRAS, 269, 785

Binggeli, B., Tammann, G. A., \& Sandage, A. 1987, AJ, 94, 251

Binney, J., \& Tremaine, S. 1994, Galactic Dynamics (Princeton: Princeton Univ. Press)

Boroson, T. A., Thompson, I. B., \& Shectman, S. 1983, AJ, 88, 1707

Brodie, J. P., \& Huchra, J. P. 1991, ApJ, 379, 157

Cohen, J. G. 1986, AJ, 92, 1039

. 1988, AJ, 95, 682

de Vaucouleurs, G., \& Nieto, J.-L. 1978, ApJ, 220, 449

de Zeeuw, T., \& Franx, M. 1991, ARA\&A, 29, 239

Fabricant, D., \& Gorenstein, P. 1983, ApJ, 267, 535

Ferrarese, L., Livio, M., Freedman, W., Saha, A., Stetson, P. B., Ford,

H. C., Hill, R. J., \& Madore, B. F. 1996, ApJ, 468, L95

Grillmair, C. J., Freeman, K. C., Bicknell, G. V., Carter, D., Couch, W. J.,

Sommer-Larsen, J., \& Taylor, K. 1994, ApJ, 422, L9

Harris, W. E. 1986, AJ, 91, 822

Hernquist, L. 1990, ApJ 356, 359

Huchra, J. P., \& Brodie, J. P. 1987, AJ, 93, 779

\section{REFERENCES}

Jarvis, B. J., \& Peletier, R. F. 1991, A\&A, 247, 315

Kent, S. M. 1987, AJ, 93, 816

Lauer, T., \& Kormendy, J. 1986, ApJ, 303, L1

Matsumoto, H., Koyama, K., Awaki, H., Tomida, H., Tsuru, T., Mushotzky, P., \& Hatsukade, I. 1996, PASJ, 48, 201

McLaughlin, D. E., Harris, W. E., \& Hanes, D. A. 1994, ApJ, 422, 486

Merritt, D. 1997, AJ, in press

Merritt, D., Meylan, G., \& Mayor, M. 1997, AJ, in press

Merritt, D., \& Tremblay, B. 1993, AJ, 106, 2229

Mould, J. R., Oke, J. B., de Zeeuw, P. T., \& Nemec, J. M. 1990, AJ, 99, 1823

Mould, J. R., Oke, J. B., \& Nemec, J. M. 1987, AJ, 93, 53

Nulsen, P. E. J., \& Böhringer, H. 1995, MNRAS, 274, 1093

Oke, J. B., et al. 1995, PASP, 107, 307

Pierce, M. J., Welch, D. L., McClure, R. D., van den Bergh, S., Racine, R., \& Stetson, P. B. 1994, Nature, 371, 385

Press, W. H., Flannery, B. P., Teukolsky, S. A., \& Vetterline, W. T. 1986,

Numerical Recipes (Cambridge: Cambridge Univ. Press)

Rogstad, D. H., \& Shostak, G. S. 1972, ApJ, 176, 315 
Rubin, V. C., Burstein, D., Ford, W. K., Jr., \& Thonnard, N. 1985, ApJ, 289,81

Saglia, R. P., Bertin, G., \& Stiavelli, M. 1992, ApJ, 384, 433

Saha, A., Sandage, A., Labhardt, L., Schengeler, H., Tammann, G. A., Panagia, N., \& Macchetto, F. D. 1995, ApJ, 438, 8

Sargent, W. L. W., Young, P. J., Boksenberg, A., Shortridge, K., Lynds, C. R., \& Hartwick, F. D. A. 1978, ApJ, 221, 731
Sembach, K. R., \& Tonry, J. L. 1996, AJ, 112, 797

Strom, S. E., Forte, J., Harris, W., Strom, K. M., Wells, D., \& Smith, M. 1981, ApJ, 245, 416

Tsai, J. C. 1993, ApJ, 413, L59

1994, ApJ, 429, 119

van der Marel, R. P. 1994, MNRAS, 270, 271 